\title{
The relativistic galaxy number counts in the weak field approximation
}

\author{
Enea Di Dio ${ }^{a, b, c}$ Florian Beutler ${ }^{d, e}$
}

${ }^{a}$ Center for Theoretical Astrophysics and Cosmology, Institute for Computational Science, University of Zurich, Winterthurerstrasse 190, CH-8057 Zurich, Switzerland

${ }^{b}$ Physics Division, Lawrence Berkeley National Laboratory, Cyclotron Rd, Berkeley, CA 94720

${ }^{c}$ Berkeley Center for Cosmological Physics and Department of Physics, University of California, Berkeley, CA 94720

${ }^{d}$ Institute for Astronomy, University of Edinburgh, Royal Observatory, Blackford Hill, Edinburgh EH9 3HJ, UK

${ }^{e}$ Institute of Cosmology \& Gravitation, University of Portsmouth, Dennis Sciama Building, Burnaby Road, Portsmouth PO1 3FX, UK

E-mail: enea.didio@uzh.ch, florian.beutler@port.ac.uk

\begin{abstract}
We present a novel approach to compute systematically the relativistic projection effects at any order in perturbation theory within the weak field approximation. In this derivation the galaxy number counts is written completely in terms of the redshift perturbation. The relativistic effects break the symmetry along the line-of-sight and they source, contrarily to the standard perturbation theory, the odd multipoles of the matter power spectrum or 2-point correlation function, providing a unique signature for their detection in Large Scale Structure surveys. We show that our approach agrees with previous derivations (up to third order) of relativistic effects and, for the first time, we derive a model for the transverse Doppler effect. Moreover, we show that in the Newtonian limit this approach is consistent with standard perturbation theory at any order.
\end{abstract}




\section{Contents}

1 Introduction 1

2 Relativistic effects 2

3 Redshift perturbation $\quad 3$

4 Density perturbation 5

5 Volume perturbation $\quad 6$

6 Full relativistic number counts $\quad 7$

6.1 Linear Perturbation Theory 8

6.2 Second order Perturbation Theory 9

6.3 Third order Perturbation Theory 9

7 Single contributions to galaxy number counts 10

$\begin{array}{ll}7.1 & \text { Gravitational potential } \\ & 10\end{array}$

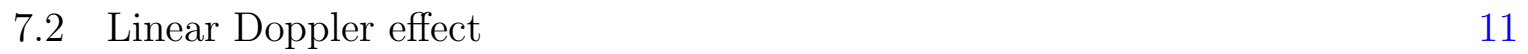

7.3 Transverse Doppler effect 11

8 Newtonian limit $\quad 12$

9 Conclusions 14

\section{Introduction}

The so-called relativistic projection effects arise form the change of coordinates required to describe galaxy redshift survey observables directly in terms of observed quantities, namely the redshift $z$ and the line-of-sight direction $\mathbf{n}$. In doing this change of coordinates we have to carefully consider that both these quantities are perturbed with respect to the background cosmology. While current derivations and expressions of relativistic corrections are long and tedious (see for instance Refs. [1-14]), one should be able to recover them directly from the shift in redshift $\delta z$ and in the line-of-sight $\delta \mathbf{n}$, at least the leading non-vanishing order in the weak field approximation.

Moreover, this approach provides a clear interpretation in terms of numerical N-body simulations. Indeed, it is worth reminding that relativistic projection effects are purely geometrical and independent of the theory of gravity. Because of that, these effects can be correctly computed using geodesic light tracing in a Newtonian N-body simulation, see e.g. Ref. [15].

Intuitively we can associate the redshift $\delta z$ and the line-of-sight $\delta \mathbf{n}$ perturbations to radial and transverse modes, respectively. The shift in transverse position $\delta \mathbf{n}$ is generated by the lensing effect of foreground structures and is a well-known effect studied and detected for several different observables: CMB (see Ref. [16] for an extensive review), magnification and shear galaxies (see reviews [17-19]), CIB [20], 21cm intensity mapping [21] and matter power spectrum [22-27]. In this work we focus on the effects generated by the redshift perturbation $\delta z$, which is sourced by peculiar motion of galaxies and 
geometrical perturbations in the form of gravitational redshift and integrated Sachs-Wolf effect (ISW). The relativistic projection effects generated by the redshift perturbation peak along the radial direction, e.g. $\mu \equiv-\mathbf{n} \cdot \hat{\mathbf{k}}= \pm 1$, where $\mathbf{k}$ is the Fourier wave vector associated with the pair separation and $\mathbf{n}$ is the line-of-sight unit vector pointing from the source to the observer. The largest effect induced by the redshift perturbation is the well-known Kaiser redshift space distortion [28]. While the latter is proportional to $\mu^{2}$, relativistic effects generate terms proportional to odd powers of $\mu$. Therefore, differently from standard perturbation theory, relativistic effects source the odd multipoles of the matter power spectrum or 2-point correlation function, as first pointed out in Ref. [29] and then studied for different observables [10, 15, 30-37].

In this work we develop a formalism to easily obtain the relativistic corrections which source the odd multipoles to any order in perturbation theory, omitting integrated terms whose magnitudes are expected to be negligible in terms of the odd multipole statistics, in most of the relevant configurations. Our work provides the necessary tools to compute the odd-multipole statistics of the power spectrum or the 2-point correlation function of the Large Scale Structures (LSS) beyond linear theory.

In an accompanying paper [38], we adopt this formalism to compute the dipole of the matter power spectrum at 1-loop in perturbation theory and compare our theoretical prediction with the relativistic N-body simulation presented in Ref. [15].

The paper is organized as follows: in Section 2 we introduce the relativistic effects within the weak field approximation and then, in Section 3, we compute the full redshift perturbation within this approximation. In Sections 4 and 5 we compute the density and the volume perturbations, respectively, sourced by the redshift perturbation $\delta z$. In Section 6 we derive the full galaxy number counts and we compare with previous derivations up to third order in perturbation theory. In Section 7 we derive the relativistic effects induced directly by the gravitational redshift, the linear and transverse Doppler in the redshift perturbation. In Section 8 we proof that in the Newtonian limit our approach fully agrees with standard perturbation theory at any order and we conclude in Section 9.

\section{Relativistic effects}

A galaxy clustering survey maps the galaxy distribution $N(\mathbf{n}, z)$ as a function of lineof-sight direction $\mathbf{n}$ and redshift $z$. From this we can define the galaxy number counts as

$$
\Delta(\mathbf{n}, z)=\frac{N(\mathbf{n}, z)-\langle N(\mathbf{n}, z)\rangle}{\langle N(\mathbf{n}, z)\rangle}
$$

where $\langle.$.$\rangle denotes the angular average at constant observed redshift z$.

Starting from a density field $\rho(t, \mathbf{x})$ we need to perform a change of coordinates to determine the galaxy number counts $\Delta(\mathbf{n}, z)$ as a function of the observed angle $\mathbf{n}$ and redshift $z$. The matter density $\rho(t, \mathbf{x})$ will transform under a change of coordinates as a density scalar field: through a density and a volume part, studied in Sections 4 and 5, respectively. This change of coordinates requires to solve the geodesic light propagation from the source to the observer. Therefore, the galaxy number counts $\Delta(\mathbf{n}, z)$ will also be affected by the matter distribution between the source and the observer. Moreover, it will also be sensitive to the peculiar motion of the source which impacts the redshift measurement. The leading part of the dependence on the peculiar motion of the source is captured by the redshift space distortion effect [28]. However, several other subleading 
contributions are not captured in the Newtonian derivation of the redshift space distortion effect and we usually refer to them as relativistic corrections.

More precisely, we decompose the galaxy number counts in a Newtonian part $\Delta_{N}$ and a (leading) relativistic correction $\Delta_{R}$ as

$$
\Delta=\Delta_{N}+\Delta_{R}+\mathcal{O}\left(\epsilon_{\mathcal{H}}\right)
$$

where the relativistic part is suppressed as

$$
\Delta_{R} \sim \epsilon_{\mathcal{H}}^{1 / 2} \Delta_{N} \quad \text { and } \quad \epsilon_{\mathcal{H}} \equiv\left(\frac{\mathcal{H}}{k}\right)^{2},
$$

where $\mathcal{H}$ is the comoving Hubble parameter and $k$ the Fourier wavelength. We remark that under this definition we do not consider lensing magnification as a relativistic effect. The impact of lensing magnification on galaxy clustering has been already extensively studied (see e.g. Refs. [22-26, 39-44]). This definition of relativistic effects indicates that corrections to the standard (Newtonian) perturbation theory could be relevant at large scales, e.g. when these corrections are less suppressed by the parameter $\epsilon_{\mathcal{H}}$. However, the detection of relativistic effects at the largest scales is strongly limited by cosmic variance. The parameter $\epsilon_{\mathcal{H}}$ corresponds to the inverse Laplacian in real space. Therefore, the relativistic $\Delta_{R}$ and the Newtonian $\Delta_{N}$ parts differ by a single spatial derivative. As pointed out before, the redshift perturbation $\delta z$ is mainly induced by radial modes. Hence, as we will see in detail in the next sections, $\Delta_{N}$ and $\Delta_{R}$ differ by a radial derivative or a factor $i \mu \mathcal{H} / k$ in Fourier space. Therefore, relativistic terms $\Delta_{R}$ differ also by a factor of $\mu$ with respect to the standard Newtonian terms $\Delta_{N}$. Since the latter are characterized only by even powers of $\mu$ (i.e. are symmetric with respect to the angle between the line-ofsight and the pair separation) the relativistic corrections are proportional to odd powers of $\mu$. For this reason, when correlating two differently biased samples of galaxies, the correlation $\left\langle\Delta_{N} \Delta_{R}\right\rangle$ sources odd multipoles of the power spectrum or 2-point correlation function.

We want to emphasise that this approach is purely geometrical and it can be applied to any metric theory of gravity. It therefore provides an appropriate framework to test gravity and the Equivalence Principle [45] at the largest scales. Furthermore, we only work within the weak field approximation neglecting terms directly proportional to the metric perturbations. Any contributions to the odd power spectrum multipoles beyond the weak field approximation are suppressed by $\epsilon_{\mathcal{H}}^{3 / 2}$.

\section{Redshift perturbation}

The first step in our approach consists in determining the redshift perturbation within the weak field approximation. We remind the reader that we do not intend to expand perturbatively around the matter density fluctuation $\delta_{m}$, which can be order 1 in our derivation. We instead assume that the metric perturbation are small at any scale, i.e. $\Psi \sim \Phi \ll 1$. We start considering a perturbed FLRW metric

$$
d s^{2}=a(t)^{2}\left[-(1+2 \Psi) d t^{2}+(1-2 \Phi)\left(d r^{2}+r^{2} d \Omega\right)\right],
$$

where $\Psi$ and $\Phi$ denote (to first order) the Bardeen potentials and $t$ is the conformal time. In an arbitrary space-time the observed redshift is defined as

$$
1+z=\frac{\left.k^{\mu} u_{\mu}\right|_{s}}{\left.k^{\mu} u_{\mu}\right|_{o}},
$$


where the suffixes $s$ and $o$ denote the source and the observer positions, respectively. The 4 -vector $k^{\mu}=d x^{\mu} / d \lambda$ is the tangent vector along the light geodesic connecting the source and the observer, determined by the geodesic equations

$$
\frac{d k^{\mu}}{d \lambda}+\Gamma_{\nu \lambda}^{\mu} k^{\nu} k^{\lambda}=0
$$

where $\Gamma_{\nu \lambda}^{\mu}$ are the Christoffel symbols associated to the metric (3.1). The 4-velocity $u^{\mu}$ is time-like and normalized such that

$$
u^{\mu} u_{\mu}=-1
$$

By writing the 4-velocity as $\left(u^{\mu}\right)=a^{-1}\left(u^{0}, v^{i}\right)$, where $\mathbf{v}$ is the 3-dimensional peculiar velocity, we can fully constrain the $u^{0}$ component from eq. (3.4)

$$
u^{0}=\sqrt{\frac{1+(1-2 \Phi) v^{2}}{1+2 \Psi}}=1+\frac{v^{2}}{2}-\Psi+\mathcal{O}\left(\epsilon_{\mathcal{H}}^{2}\right),
$$

where we have expanded in the weak field parameter ${ }^{1} \epsilon_{\mathcal{H}}$. Within this scheme ${ }^{2}$ we have $\Psi \sim \Phi \sim v^{2} \sim \epsilon_{\mathcal{H}} \ll 1$. This approximation holds even after shell crossing in structure formation. Indeed the collapse of structures is counteracted by the virialized motion, which predicts $\Psi \sim \Phi \sim v^{2}$. While the amplitude of over-densities becomes hundred times the mean density of the universe in virialized object, the gravitational potentials are small everywhere and they become order unity only in the proximity of black holes.

By using the solution to the geodesic eq. (3.3)

$$
\delta k^{0}-\delta k_{o}^{0}=2 \Psi_{o}-2 \Psi+\mathcal{O}\left(\epsilon_{\mathcal{H}}^{2}\right)
$$

where we have neglected the integrated Sachs-Wolfe (ISW) contribution and the suffix $o$ denotes the quantities evaluated at the observer position, we obtain the observed redshift

$$
1+z=(1+\bar{z})\left(1+\left[-v_{\|}-\Psi+\frac{v^{2}}{2}\right]_{o}^{\bar{z}}\right)+\mathcal{O}\left(\epsilon_{\mathcal{H}}^{3 / 2}\right)
$$

where we have introduced the background redshift $\bar{z}$ through $1+\bar{z}=1 / a(t)$.

The redshift perturbation is defined as

$$
\delta z=z-\bar{z} .
$$

By plug in the redshift perturbation $\delta z$ and Taylor expanding around the observed redshift $z$, under the assumption $\delta z \ll 1$ we obtain

$$
\begin{aligned}
\delta z & =(1+\bar{z})\left[\frac{v^{2}}{2}-v_{\|}-\Psi\right]_{o}^{\bar{z}=z-\delta z}+\mathcal{O}\left(\epsilon_{\mathcal{H}}^{3 / 2}\right) \\
& =\sum_{i=0} \frac{1}{i !}(-\delta z)^{i} \frac{d^{i}}{d z^{i}}\left[(1+z)\left(\frac{v^{2}}{2}-v_{\|}-\Psi-\frac{v_{o}^{2}}{2}+v_{\|_{o}}+\Psi_{o}\right)\right]+\mathcal{O}\left(\epsilon_{\mathcal{H}}^{3 / 2}\right) .
\end{aligned}
$$

\footnotetext{
${ }^{1}$ For sake of simplicity we always denote the weak field parameter $\epsilon_{\mathcal{H}}$ in Fourier space. As specified before, in real space the weak field parameter $\epsilon_{\mathcal{H}}$ is related to the inverse of the Laplacian operator.

${ }^{2}$ This scheme has been theoretical motivated in Refs. [46, 47], and later adopted in several other works, see for instance Refs. [48-52].
} 
where we have considered ${ }^{3} \frac{d^{i}}{d z^{i}} \sim \epsilon_{\mathcal{H}}^{-i / 2}$ and $\delta z \sim \epsilon_{\mathcal{H}}^{1 / 2}$. We now make the following Ansatz

$$
\delta z=\left.\sum_{i} \delta z\right|_{i} \quad \text { where }\left.\quad \delta z\right|_{i}=\mathcal{O}\left(\left[(1+z)\left(\frac{v^{2}}{2}-v_{\|}-\Psi-\frac{v_{o}^{2}}{2}+v_{\|_{o}}+\Psi_{o}\right)\right]^{i}\right) .
$$

With this Ansatz and eq. (3.9) we can determine any term $\left.\delta z\right|_{i}$ as a function of all other contributions $\left.\delta z\right|_{j}$ with $j<i$. For instance we have

$$
\begin{aligned}
& \left.\delta z\right|_{1}=(1+z)\left(\frac{v^{2}}{2}-v_{\|}-\Psi-\frac{v_{o}^{2}}{2}+v_{\|_{o}}+\Psi_{o}\right), \\
& \left.\delta z\right|_{2}=-\left.\delta z\right|_{1} \frac{\left.d \delta z\right|_{1}}{d z}, \\
& \left.\delta z\right|_{3}=-\left.\delta z\right|_{2} \frac{\left.d \delta z\right|_{1}}{d z}+\frac{1}{2}\left(\left.\delta z\right|_{1}\right)^{2} \frac{\left.d^{2} \delta z\right|_{1}}{d z^{2}}, \\
& \left.\delta z\right|_{4}=-\left.\delta z\right|_{3} \frac{\left.d \delta z\right|_{1}}{d z}+\left.\left.\delta z\right|_{1} \delta z\right|_{2} \frac{\left.d^{2} \delta z\right|_{1}}{d z^{2}}-\frac{1}{6}\left(\left.\delta z\right|_{1}\right)^{3} \frac{\left.d^{3} \delta z\right|_{1}}{d z^{3}} .
\end{aligned}
$$

Moreover, we can re-sum all the contributions $\left.\delta z\right|_{i}$, such that we can write the full redshift perturbation as

$$
\delta z=-\sum_{i=0} \frac{1}{(i+1) !} \frac{d^{i}}{d z^{i}}\left[(1+z)\left(\Psi+v_{\|}-\frac{v^{2}}{2}-\Psi_{o}-v_{\|_{o}}+\frac{v_{o}^{2}}{2}\right)\right]^{i+1}+\mathcal{O}\left(\epsilon_{\mathcal{H}}^{3 / 2}\right),
$$

which satisfies eq. (3.9). The three contributions to the redshift-space distortion are due to gravitational redshift as well as the linear and transverse Doppler effect. Clearly, beyond linear theory these three terms are coupled together.

\section{Density perturbation}

Now that we have derived the redshift perturbation $\delta z$, we want to study its relation to the observed galaxy density. In this section we are therefore interested in the relativistic corrections to the density part of the galaxy number counts. We start considering the observed density $\rho_{\text {obs }}(z, \mathbf{n})$ as a function of the measured redshift $z$ and photon direction n. This is related to the theoretical density $\rho$ as

$$
\rho_{\text {obs }}(z, \mathbf{n})=\rho_{\text {obs }}\left(\bar{z}+\delta z, \mathbf{n}^{(0)}+\delta \mathbf{n}\right)=\rho\left(\bar{z}, \mathbf{n}^{(0)}\right) .
$$

Since the perturbations of the photon direction are due to the deflection angle sourced by the lensing potential, we neglect its contribution along this derivation as motivated in the previous sections. A non-perturbative study of the impact of the deflection angle to the matter power spectrum [27] and on the correlation function [25] have been already performed.

In order to express the density perturbation as a function of the observed redshift $z$ we simply need to Taylor expand eq. (4.1)

$$
\delta_{z}=\sum_{i} \frac{1}{\bar{\rho}} \frac{1}{i !} \frac{d^{i}}{d z^{i}}[\bar{\rho}(1+\delta)](-\delta z)^{i}-1
$$

\footnotetext{
${ }^{3}$ Since $\frac{d A(t, r)}{d z}=\frac{d t}{d z} \partial_{t} A+\frac{d r}{d z} \partial_{r} A$, in the redshift derivative there is also a contribution of the order $\epsilon_{\mathcal{H}}^{0}$, however this is subdominant with respect to the radial derivative which is of the order $\epsilon_{\mathcal{H}}^{-1 / 2}$. Therefore considering $\frac{d^{i}}{d z^{i}} \sim \epsilon_{\mathcal{H}}^{-i / 2}$ is correct up to subleading terms in our perturbative expansion.
} 
where we have rewritten $\rho=\bar{\rho}(1+\delta)$. Being the redshift perturbation $\delta z$ of the order $\epsilon^{1 / 2}$ we can approximate $\delta_{z}$ through

$$
\begin{aligned}
\delta_{z}= & \sum_{i=0} \frac{1}{i !}\left(\frac{-\delta z}{\mathcal{H}(1+z)}\right)^{i}\left[\partial_{r}^{i}+\mathcal{H} i\left(\frac{1-i}{2}\left(1-\frac{\dot{\mathcal{H}}}{\mathcal{H}^{2}}\right)+3-b_{e}\right) \partial_{r}^{i-1}-i \partial_{r}^{i-1} \partial_{t}\right] \delta \\
& +\mathcal{O}\left(\epsilon_{\mathcal{H}}\right),
\end{aligned}
$$

where we have introduced the evolution bias (see e.g. $[4,53]$ )

$$
b_{e}=3-(1+z) \frac{d \ln \bar{\rho}}{d z}
$$

to account for different galaxy evolution.

A non-vanishing evolution bias indicates that the number of sources is not conserved in a comoving volume. Clearly for Dark Matter, $\rho_{D M} \propto a(t)^{-3}$, we have $b_{e}=0$. However biased tracers may have a different background density evolution. Interestingly, while $b_{e}$ is a function of redshift, its derivative does not contribute to the (leading) relativistic number counts. Indeed the term $b_{\text {evo }}^{\prime}(z)$ is associated with the second derivative of the background density. However, acting in eq. (4.2) with more than one redshift derivative on the background density $\bar{\rho}$, it reduces the number of spatial derivatives on the density perturbation $\delta$, suppressing these terms beyond the weak field approximation.

\section{Volume perturbation}

We now consider the contribution due to the volume perturbation. We follow the derivation of Ref. [3] by considering the following volume element around a source with 4velocity given by (3.5)

$$
d V=\sqrt{-g} \epsilon_{\mu \nu \alpha \beta} u^{\mu} d x^{\nu} d x^{\alpha} d x^{\beta}=v(z, \theta, \varphi) d z d \theta d \varphi,
$$

where $\epsilon_{\mu \nu \alpha \beta}$ is the Levi-Civita anti-symmetric tensor. In line with the derivation of Ref. [12] we have

$$
\begin{aligned}
v_{\text {obs }}(z, \theta \varphi) & =\sqrt{-g} \epsilon_{\mu \nu \alpha \beta} u^{\mu} \frac{\partial x^{\nu}}{\partial z} \frac{\partial x^{\alpha}}{\partial \theta} \frac{\partial x^{\beta}}{\partial \varphi} \\
& =\left[a^{3} r^{2} \sin \theta\right](\bar{z})\left\{\left(1+\frac{v^{2}}{2}-\Psi\right) \frac{d r(\bar{z})}{d z}+v_{\|}(\bar{z}) \frac{d t(\bar{z})}{d z}\right\}+\mathcal{O}\left(\epsilon_{\mathcal{H}}\right) \\
& =\left[a^{3} r^{2} \sin \theta\right](\bar{z}) \frac{d r}{d \bar{z}}\left(1-\frac{d \delta z}{d z}\right)\left(1+\frac{v^{2}}{2}-\Psi-v_{\|}\right)_{z=\bar{z}}+\mathcal{O}\left(\epsilon_{\mathcal{H}}\right) \\
& =\left[\frac{a^{4} r^{2} \sin \theta}{\mathcal{H}}\right](\bar{z})\left(1-\frac{d \delta z}{d z}\right)\left(1+\frac{\delta z}{1+z}-v_{\|_{o}}\right)+\mathcal{O}\left(\epsilon_{\mathcal{H}}\right) \\
& =\bar{v}\left(1-\frac{1}{\bar{v}} \frac{d \bar{v}}{d z} \delta z\right)\left(1-\frac{d \delta z}{d z}\right)\left(1+\frac{\delta z}{1+z}-v_{\|_{o}}\right)+\mathcal{O}\left(\epsilon_{\mathcal{H}}\right),
\end{aligned}
$$

where we have introduced the background volume element $\bar{v}=r^{2} a^{4} \sin \theta / \mathcal{H}$. Consistent with the previous approximation we have neglected the contribution due to angular Jacobian

$$
\left|\frac{\partial\left(\theta_{s}, \varphi_{s}\right)}{\partial\left(\theta_{o}, \varphi_{o}\right)}\right|
$$

Indeed, this leads to lensing effects, which we are neglecting in this approach. 


\section{$6 \quad$ Full relativistic number counts}

Once having derived the density and the volume contributions to the number counts, we can simply combine them to get the full relativistic number counts as function of the redshift perturbation $\delta z$

$$
\begin{aligned}
\Delta(\mathbf{n}, z)= & \left(1+\delta_{z}\right) \frac{v_{\mathrm{obs}}}{\bar{v}}-1= \\
= & \sum_{i=0} \frac{1}{i !}\left(\frac{-\delta z}{\mathcal{H}(1+z)}\right)^{i}\left[\partial_{r}^{i}+\mathcal{H} i\left(\frac{1-i}{2}\left(1-\frac{\dot{\mathcal{H}}}{\mathcal{H}^{2}}\right)+3-b_{e}\right) \partial_{r}^{i-1}\right. \\
& \left.\quad-i \partial_{r}^{i-1} \partial_{t}\right](1+\delta) \\
& \times\left(1-\frac{1}{\bar{v}} \frac{d \bar{v}}{d z} \delta z\right)\left(1-\frac{d \delta z}{d z}\right)\left(1+\frac{\delta z}{1+z}-v_{\|_{o}}\right)-1+\mathcal{O}\left(\epsilon_{\mathcal{H}}\right) .
\end{aligned}
$$

We stress, once again, that this expression is valid at any order in perturbation theory. We clearly see that it depends completely on the redshift perturbation $\delta z$.

We can further simplify this expression by decomposing the redshift perturbation in a Newtonian and a relativistic part

$$
\delta z=\delta z_{N}+\delta z_{R}+\mathcal{O}\left(\epsilon_{\mathcal{H}}^{3 / 2}\right), \quad \text { where } \quad \delta z_{R} \sim \epsilon^{1 / 2} \delta z_{N}
$$

In this case, neglecting the terms evaluated at the observer position for sake of simplicity, we obtain

$$
\begin{aligned}
& \delta z_{N}=-(1+z) \sum_{i=0} \frac{\mathcal{H}^{-i}}{(1+i) !} \partial_{r}^{i} v_{\|}^{i+1} \\
& \delta z_{R}=-(1+z) \sum_{i=0} \frac{\mathcal{H}^{1-i}}{(i+1) !}\left[\frac{i}{2}\left(i+3+(i-1) \frac{\dot{\mathcal{H}}}{\mathcal{H}^{2}}\right) \partial_{r}^{i-1} v_{\|}^{i+1}\right. \\
&\left.\quad-\frac{i(i+1)}{\mathcal{H}} \partial_{r}^{i-1}\left(v_{\|}^{i} \dot{v}_{\|}\right)+\frac{i+1}{\mathcal{H}} \partial_{r}^{i}\left(v_{\|}^{i}\left(\Psi-\frac{v^{2}}{2}\right)\right)\right] .
\end{aligned}
$$

By adopting this redshift decomposition we get

$$
\begin{aligned}
\Delta(\mathbf{n}, z)= & \left(1-\frac{d \delta z}{d z}\right)\left\{\sum_{i=0} \frac{1}{i !}\left(\frac{-\delta z}{\mathcal{H}(1+z)}\right)^{i} \partial_{r}^{i}(1+\delta)\right. \\
& \left.+\sum_{i=1} \frac{1}{i !}\left(\frac{-\delta z_{N}}{\mathcal{H}(1+z)}\right)^{i}\left[\mathcal{H} i\left(\frac{1-i}{2}\left(1-\frac{\dot{\mathcal{H}}}{\mathcal{H}^{2}}\right)+3-b_{e}\right) \partial_{r}^{i-1}-i \partial_{r}^{i-1} \partial_{t}\right] \delta\right\} \\
& \times\left(1-\frac{1}{\bar{v}} \frac{d \bar{v}}{d z} \delta z_{N}+\frac{\delta z_{N}}{1+z}\right)-1+\mathcal{O}\left(\epsilon_{\mathcal{H}}\right) .
\end{aligned}
$$

In the next sections we will show briefly that we recover correctly the known results up to third order in perturbation theory with this simple approach. As shown in eq. (6.1), our new approach can easily obtain the (leading) relativistic perturbation to higher orders in perturbation theory without the need to re-derive them from scratch at any perturbative order. 


\subsection{Linear Perturbation Theory}

In this section we will show that our approach does recover the well-known number count at linear order. Within linear theory we can neglect the transverse Doppler effect and therefore the redshift perturbation is simply given by ${ }^{4}$

$$
\begin{aligned}
\delta z & =-\sum_{i=0} \frac{1}{(i+1) !} \frac{d^{i}}{d z^{i}}\left[(1+z)\left(\Psi+v_{\|}-\frac{v^{2}}{2}-v_{\|_{o}}-\Psi_{o}+\frac{v_{o}^{2}}{2}\right)\right]^{i+1}+\mathcal{O}\left(\epsilon_{\mathcal{H}}^{3 / 2}\right) \\
& =-(1+z)\left(\Psi+v_{\|}-\Psi_{o}-v_{\|_{o}}\right)+\mathcal{O}\left(\epsilon_{\mathcal{H}}^{3 / 2}\right),
\end{aligned}
$$

and in particular

$$
\begin{aligned}
& \delta z_{N}=-(1+z)\left(v_{\|}-v_{\|_{o}}\right) \\
& \delta z_{R}=-(1+z)\left(\Psi-\Psi_{o}\right) .
\end{aligned}
$$

The number counts read as

$$
\begin{aligned}
\Delta^{(1)}(\mathbf{n}, z) & =\left(1+\delta-\frac{\delta z_{N}}{(1+z)}\left(3-b_{e}\right)\right)\left(1-\frac{1}{\bar{v}} \frac{d \bar{v}}{d z} \delta z_{N}-\frac{d \delta z}{d z}+\frac{\delta z_{N}}{1+z}-v_{\|_{o}}\right)-1+\mathcal{O}\left(\epsilon_{\mathcal{H}}\right) \\
& =\delta+\frac{\delta z_{N}}{1+z}\left(1-3+b_{e}-\frac{1+z}{\bar{v}} \frac{d \bar{v}}{d v}\right)-\frac{d \delta z}{d z}-v_{\|_{o}}+\mathcal{O}\left(\epsilon_{\mathcal{H}}\right) \\
& =\delta-\left(v_{\|}-v_{\|_{o}}\right)\left(2+b_{e}-\frac{\dot{\mathcal{H}}}{\mathcal{H}^{2}}-\frac{2}{\mathcal{H} r}\right)+v_{\|}+(1+z) \frac{d\left(v_{\|}+\Psi\right)}{d z}-v_{\|_{o}}+\mathcal{O}\left(\epsilon_{\mathcal{H}}\right) \\
& =\delta+\left(v_{\|}-v_{\|_{o}}\right)\left(\frac{\dot{\mathcal{H}}}{\mathcal{H}^{2}}+\frac{2}{\mathcal{H} r}-1-b_{e}\right)+\mathcal{H}^{-1}\left(\partial_{r} v_{\|}+\partial_{r} \Psi-\dot{v}_{\|}\right)-v_{\|_{o}}+\mathcal{O}\left(\epsilon_{\mathcal{H}}\right)
\end{aligned}
$$

where we have used

$$
\frac{1+z}{\bar{v}} \frac{d \bar{v}}{d v}=\frac{\dot{\mathcal{H}}}{\mathcal{H}^{2}}+\frac{2}{\mathcal{H} r}-4
$$

Considering the Euler equation

$$
\dot{v}_{\|}+\mathcal{H} v_{\|}-\partial_{r} \Psi=0
$$

we recover the well-known relativistic number counts to linear order, first derived in Refs. [1-4], namely

$$
\Delta^{(1)}(\mathbf{n}, z)=\delta+\mathcal{H}^{-1} \partial_{r} v_{\|}+\left(v_{\|}-v_{\|_{o}}\right)\left(\frac{\dot{\mathcal{H}}}{\mathcal{H}^{2}}+\frac{2}{\mathcal{H} r}-b_{e}\right)+\mathcal{O}\left(\epsilon_{\mathcal{H}}\right) .
$$

In our derivation the density fluctuation $\delta$ is defined in Newtonian gauge. In most of other references it is written in terms of the density fluctuation in synchronous comoving gauge. Being the difference between the density fluctuation defined in the two gauges of the order $\mathcal{O}\left(\epsilon_{\mathcal{H}}\right)$ we can simply exchange them in our expression. The synchronous gauge offers also a more natural framework to include the galaxy bias, see for instance [54, 55].

\footnotetext{
${ }^{4}$ The equations shown and derived in these sections are valid at the perturbative order considered. For sake of simplicity we do not explicitly denote the perturbative order in each equation. This helps also to avoid any possible confusion with the weak field approximation expressed in terms of power of $\epsilon \mathcal{H}$.
} 


\subsection{Second order Perturbation Theory}

At second order the redshift perturbation reads $\mathrm{as}^{5}$

$$
\delta z^{(2)}=\delta z^{(1 \rightarrow 2)}+(1+z)\left(\frac{v^{2}}{2}-v_{\|}^{2}-\mathcal{H}^{-1} \partial_{r}\left(v_{\|} \Psi\right)-\mathcal{H}^{-1} v_{\|} \partial_{r} v_{\|}+\mathcal{H}^{-1} v_{\|} \dot{v}_{\|}\right)+\mathcal{O}\left(\epsilon_{\mathcal{H}}^{3 / 2}\right)
$$

where $\delta z^{(1 \rightarrow 2)}$ denotes the terms which appear already at first order, simply evaluated at second order. Indeed at any order we will need to include the same operators which appear to lower orders, simply evaluated at higher order in perturbation theory, e.g. $\delta^{(2)}, v^{(2)}, \Psi^{(2)}$. We can further decompose the second order redshift perturbation in

$$
\begin{aligned}
& \delta z_{N}^{(2)}=\delta z_{N}^{(1 \rightarrow 2)}-(1+z) \mathcal{H}^{-1} v_{\|} \partial_{r} v_{\|}, \\
& \delta z_{R}^{(2)}=\delta z_{R}^{(1 \rightarrow 2)}+(1+z)\left(\frac{v^{2}}{2}-v_{\|}^{2}-\mathcal{H}^{-1} \partial_{r}\left(v_{\|} \Psi\right)+\mathcal{H}^{-1} v_{\|} \dot{v}_{\|}\right) .
\end{aligned}
$$

By using eq. (6.5) we obtain directly

$$
\begin{aligned}
\Delta_{N}^{(2)}(\mathbf{n}, z)= & \delta_{g}^{(2)}+\mathcal{H}^{-1} \partial_{r} v_{\|}^{(2)}+\mathcal{H}^{-1} \partial_{r}\left(v_{\|} \delta_{g}\right)+\mathcal{H}^{-2} \partial_{r}\left(v_{\|} \partial_{r} v_{\|}\right) \\
\Delta_{R}^{(2)}(\mathbf{n}, z)= & \left(-1+\frac{\dot{\mathcal{H}}}{\mathcal{H}^{2}}+\frac{2}{\mathcal{H} r}-b_{e}\right)\left(v_{\|}^{(2)}+v_{\|} \delta\right)-\mathcal{H}^{-1} \dot{v}_{\|}^{(2)}-2 \mathcal{H}^{-2} \partial_{r} v_{\|} \dot{v}_{\|} \\
& +\left(-2+3 \frac{\dot{\mathcal{H}}}{\mathcal{H}^{2}}+\frac{4}{\mathcal{H} r}-2 b_{e}\right) \mathcal{H}^{-1} v_{\| \mid} \partial_{r} v_{\|}-2 \mathcal{H}^{-2} v_{\|} \partial_{r} \dot{v}_{\|} \\
& -\mathcal{H}^{-1} \dot{v}_{\|} \delta-\mathcal{H}^{-1} v_{\|} \dot{\delta}+\mathcal{H}^{-1} v^{a} \partial_{a} v_{\|}+\mathcal{H}^{-2} \Psi \partial_{r}^{2} v_{\|}+\mathcal{H}^{-1} \Psi \partial_{r} \delta \\
& +\mathcal{H}^{-1} \partial_{r} \Psi^{(2)}+2 \mathcal{H}^{-2} \partial_{r} v_{\|} \partial_{r} \Psi+\mathcal{H}^{-1} \delta \partial_{r} \Psi+\mathcal{H}^{-2} v_{\|} \partial_{r}^{2} \Psi .
\end{aligned}
$$

This simple approach fully agrees with the derivations of Refs. $[9,12,13]$.

\subsection{Third order Perturbation Theory}

Similar to previous sections, we show here how to obtain straightforwardly third order perturbation theory in a relativistic framework. Again, the key quantity to derive is the redshift perturbation

$$
\begin{aligned}
\delta z_{N}^{(3)}=\delta z_{N}^{(2 \rightarrow 3)}-\frac{1+z}{6 \mathcal{H}^{2}} \partial_{r}^{2} v_{\|}^{3} & \\
\delta z_{R}^{(3)}=\delta z_{R}^{(2 \rightarrow 3)}+(1+z) & {\left[\mathcal{H}^{-2} \partial_{r}\left(\dot{v}_{\|} v_{\|}^{2}\right)-2 \mathcal{H}^{-2} \partial_{r}^{2}\left(v_{\|}^{2} \Psi\right)+2 \mathcal{H}^{-1} \partial_{r}\left(v_{\|} v^{2}\right)\right.} \\
& \left.-\left(\frac{5}{2}+\frac{\dot{\mathcal{H}}}{2 \mathcal{H}^{2}}\right) \mathcal{H}^{-1} \partial_{r} v_{\|} v_{\|}^{2}\right]
\end{aligned}
$$

Once we have derived the redshift perturbation we can plug it in eq. (6.5) to obtain

$$
\Delta_{N}^{(3)}(\mathbf{n}, z)=\delta_{g}^{(3)}+\frac{\partial_{r} v_{\|}^{(3)}}{\mathcal{H}}+\left[\mathcal{H}^{-1} \partial_{r}\left(v_{\|} \delta_{g}\right)\right]^{(3)}+\left[\mathcal{H}^{-2} \partial_{r}\left(v_{\|} \partial_{r} v_{\|}\right)\right]^{(3)}
$$

\footnotetext{
${ }^{5}$ For the rest of the paper we will neglect the terms evaluated at the observer position to simplify the comparison with previous results in the literature.
} 


$$
\begin{aligned}
&+ \frac{1}{6} \mathcal{H}^{-3} \partial_{r}^{3} v_{\|}^{3}+\frac{1}{2} \mathcal{H}^{-2} \partial_{r}^{2}\left(\delta_{g} v_{\|}^{2}\right) \\
& \Delta_{R}^{(3)}(\mathbf{n}, z)=\left\{\left(-1+\frac{\dot{\mathcal{H}}}{\mathcal{H}^{2}}+\frac{2}{\mathcal{H} r}-b_{e}\right)\left(v_{\|}^{(3)}+\left[v_{\|} \delta\right]^{(3)}\right)-\mathcal{H}^{-1} \dot{v}_{\|}^{(3)}\right. \\
&-2 \mathcal{H}^{-2}\left[\partial_{r} v_{\|} \dot{v}_{\|}\right]^{(3)}+\left(-2+3 \frac{\dot{\mathcal{H}}}{\mathcal{H}^{2}}+\frac{4}{\mathcal{H} r}-2 b_{e}\right)\left[\mathcal{H}^{-1} v_{\|} \partial_{r} v_{\|}\right]^{(3)} \\
&-2 \mathcal{H}^{-2}\left[v_{\|} \partial_{r} \dot{v}_{\|]}\right]^{(3)}-\mathcal{H}^{-1}\left[\dot{v}_{\|} \delta\right]^{(3)}-\mathcal{H}^{-1}\left[v_{\|} \dot{\delta}\right]^{(3)}+\mathcal{H}^{-1}\left[v^{a} \partial_{a} v_{\|}\right]^{(3)} \\
&+\mathcal{H}^{-2}\left[\Psi \partial_{r}^{2} v_{\|}\right]^{(3)}+\mathcal{H}^{-1}\left[\Psi \partial_{r} \delta\right]^{(3)}+\mathcal{H}^{-1} \partial_{r} \Psi^{(3)}+2 \mathcal{H}^{-2}\left[\partial_{r} v_{\|} \partial_{r} \Psi\right]^{(3)} \\
&\left.+\mathcal{H}^{-1}\left[\delta \partial_{r} \Psi\right]^{(3)}+\mathcal{H}^{-2}\left[v_{\|} \partial_{r}^{2} \Psi\right]^{(3)}\right\} \\
&+\frac{1}{2 \mathcal{H}^{3}} \partial_{r}^{3}\left(v_{\|}^{2} \Psi\right)-\frac{1}{2 \mathcal{H}^{3}} \partial_{t} \partial_{r}^{2} v_{\|}^{3}+\frac{1}{\mathcal{H}^{2}} \partial_{r}^{2}\left(\Psi v_{\|} \delta\right)-\frac{1}{2 \mathcal{H}^{2}} \partial_{r}^{2}\left(v_{\|} v^{2}\right) \\
&-\frac{1}{\mathcal{H}^{2}} \partial_{t} \partial_{r}\left(\delta v_{\|}^{2}\right)-\frac{1}{2 \mathcal{H}} \partial_{r}\left(\delta v^{2}\right)+\frac{1}{\mathcal{H}^{2}} \partial_{r}\left(\partial_{r} v_{\|} v_{\|}^{2}\right)\left(3 \frac{\mathcal{H}_{\mathcal{H}}}{\mathcal{H}^{2}}+\frac{3}{\mathcal{H} r}-\frac{3}{2} b_{e}\right) \\
&+\frac{1}{\mathcal{H}} \partial_{r}\left(\delta v_{\|}^{2}\right)\left(-\frac{1}{2}+\frac{3}{2} \frac{\dot{\mathcal{H}}}{\mathcal{H}^{2}}+\frac{2}{\mathcal{H} r}-b_{e}\right) .
\end{aligned}
$$

The terms in the curly brackets are the operators already present in $\Delta_{R}^{(2)}(\mathbf{n}, z)$ simply evaluated at third order. This result fully agrees with Ref. [12].

\section{Single contributions to galaxy number counts}

So far we have shown that our new formalism agrees with the current literature. In this section we want to fully leverage this formalism by deriving the contributions induced by the different effects of the redshift perturbation on the galaxy number counts. Being interested in testing this approach at the next-to-leading order we derive the effect on the galaxy number counts up to third order in perturbation theory. The purpose of this section is also to derive the terms that allow us to compute (in the accompanying paper [38]) the dipole induced by these effects and then compare with the RayGalGroup simulation [15]. In this regard, we do not compute the contribution of the single terms alone, but we will follow the same redshift perturbation definitions adopted in Ref. [15].

\subsection{Gravitational potential}

We start by considering the redshift perturbation induced by the gravitational potential, i.e. the so-called gravitational redshift

$$
\delta z_{1}=-(1+z) \Psi
$$

Clearly in this case we do not have any Newtonian contribution to the redshift perturbation and therefore eq. (6.5) simply reduces as (considering only terms up to third order in perturbation theory)

$$
\Delta_{1}(\mathbf{n}, z)=\left(1-\frac{d \delta z_{1}}{d z}\right)\left\{\sum_{i=0} \frac{1}{i !}\left(\frac{-\delta z_{1}}{\mathcal{H}(1+z)}\right)^{i} \partial_{r}^{i}(1+\delta)\right\}-1+\mathcal{O}\left(\epsilon_{\mathcal{H}}\right)
$$




$$
\begin{aligned}
& \simeq\left(1+\mathcal{H}^{-1} \partial_{r} \Psi\right)\left(1+\delta+\mathcal{H}^{-1} \Psi \partial_{r} \delta\right)-1+\mathcal{O}\left(\epsilon_{\mathcal{H}}\right) \\
& \simeq \delta+\mathcal{H}^{-1} \partial_{r} \Psi+\mathcal{H}^{-1} \delta \partial_{r} \Psi+\mathcal{H}^{-1} \Psi \partial_{r} \delta+\mathcal{O}\left(\epsilon_{\mathcal{H}}\right) .
\end{aligned}
$$

At the different orders in perturbation theory we have therefore

$$
\begin{aligned}
& \Delta_{1}^{(1)}(\mathbf{n}, z)=\delta_{g}+\mathcal{H}^{-1} \partial_{r} \Psi \\
& \Delta_{1}^{(2)}(\mathbf{n}, z)=\Delta_{1}^{(1 \rightarrow 2)}+\mathcal{H}^{-1} \partial_{r}\left(\delta_{g} \Psi\right) \\
& \Delta_{1}^{(3)}(\mathbf{n}, z)=\Delta_{1}^{(2 \rightarrow 3)}
\end{aligned}
$$

where the perturbations on the right-hand side are evaluated at linear order if not specified otherwise.

\subsection{Linear Doppler effect}

We now include the linear Doppler effect in redshift perturbation on top of the gravitational potential computed in the previous section

$$
\delta z_{2}=-(1+z)\left(v_{\|}+\Psi\right)
$$

Since now we also have a non-vanishing Newtonian redshift perturbation sourced by the linear Doppler effect the derivation is slightly more involving. By plugging the redshift perturbation $\delta z_{2}$ in eq. (6.5) we finally obtain

$$
\begin{aligned}
\Delta_{2}^{(1)}(\mathbf{n}, z)= & \delta_{g}+\mathcal{H}^{-1} \partial_{r} v_{\|}+\mathcal{H}^{-1} \partial_{r} \Psi-\mathcal{H}^{-1} \dot{v}_{\|}+v_{\|} \mathcal{R} \\
\Delta_{2}^{(2)}(\mathbf{n}, z)= & \Delta_{2}^{(1 \rightarrow 2)}+\mathcal{H}^{-1} \partial_{r}\left(\delta_{g}\left(\Psi+v_{\|}\right)\right)-\mathcal{H}^{-1} \partial_{t}\left(\delta_{g} v_{\|}\right)+\delta_{g} v_{\|} \mathcal{R} \\
& +\mathcal{H}^{-1} v_{\|} \partial_{r} v_{\|}(\mathcal{R}-1) \\
\Delta_{2}^{(3)}(\mathbf{n}, z)= & \Delta_{2}^{(2 \rightarrow 3)}+\mathcal{H}^{-1} \delta_{g} v_{\|} \partial_{r} v_{\|}(\mathcal{R}-1)+\mathcal{H}^{-2} \partial_{r}\left(v_{\|} \Psi \partial_{r} \delta_{g}\right)-\mathcal{H}^{-2} \partial_{r}\left(v_{\|} \dot{\delta}_{g}\right) v_{\|} \\
& -\mathcal{H}^{-2} \partial_{r} \delta_{g} \dot{v}_{\|} v_{\|}+\frac{\mathcal{H}^{-1}}{2} v_{\|}^{2} \partial_{r} \delta_{g}\left(b_{e}+3 \mathcal{R}-\frac{2}{\mathcal{H} r}\right)
\end{aligned}
$$

where we have introduced the parameter

$$
\mathcal{R}=-1-b_{e}+\frac{\dot{\mathcal{H}}}{\mathcal{H}^{2}}+\frac{2}{\mathcal{H} r}
$$

Since the galaxy number counts do not depend linearly on the redshift perturbation we obtain a coupling between the Doppler $v_{\|}$and the gravitational potential $\Psi$ at third order in perturbation theory.

\subsection{Transverse Doppler effect}

We now include also the last effect which sources the redshift perturbation, namely the transverse Doppler term

$$
\delta z_{3}=-(1+z)\left(v_{\|}+\Psi-\frac{v^{2}}{2}\right)
$$

Similarly to the previous section we plug the redshift perturbation $\delta z_{3}$ into eq. (6.5) obtaining

$$
\Delta_{3}^{(1)}(\mathbf{n}, z)=\Delta_{2}^{(1)},
$$




$$
\begin{aligned}
& \Delta_{3}^{(1)}(\mathbf{n}, z)=\Delta_{2}^{(2)}-\mathcal{H}^{-1} \mathbf{v} \cdot \partial_{r} \mathbf{v} \\
& \Delta_{3}^{(1)}(\mathbf{n}, z)=\Delta_{2}^{(3)}-\mathcal{H}^{-1}\left[\mathbf{v} \cdot \partial_{r} \mathbf{v}\right]^{(3)}-\frac{\mathcal{H}^{-1}}{2} \partial_{r}\left(\delta_{g} v^{2}\right)
\end{aligned}
$$

Since the transverse Doppler effect is a second order effect, the linear galaxy number counts fully agree with the one derived in the previous section for $\delta z_{2}$.

\section{Newtonian limit}

In this section we show that the formalism, we developed in this manuscript, recovers non-perturbatively the correct Newtonian limit. In previous sections we have already shown this up to third order in perturbation theory. In the Newtonian limit we have

$$
\Delta_{N}(\mathbf{n}, z)=\left(\sum_{i=0} \frac{1}{i !}\left(\frac{-\delta z_{N}}{\mathcal{H}(1+z)}\right)^{i} \partial_{r}^{i}(1+\delta)\right)\left(1-\frac{d \delta z_{N}}{d z}\right)-1 .
$$

We aim to show that this equation agrees with (see Ref. [56])

$$
\delta_{s}(\mathbf{x})=\sum_{L=0} \frac{1}{L !} \mathcal{H}^{-L} \partial_{r}^{L}\left[(1+\delta(\mathbf{x})) v_{\|}(\mathbf{x})^{L}\right] .
$$

We can re-express this result in terms of sum over different perturbative order $n$

$$
\delta_{s}(\mathbf{x})=\sum_{n=0} \frac{1}{n !} \mathcal{H}^{-n} \partial_{r}^{n} v_{\|}^{n}+\sum_{n=0} \frac{1}{(n-1) !} \mathcal{H}^{-n+1} \partial_{r}^{n-1}\left(\delta v_{\|}^{n-1}\right) .
$$

Then rewriting eq. (8.1) as

$$
\begin{aligned}
\Delta_{N}(\mathbf{n}, z) & =\left(1+\sum_{i=0} \frac{1}{i !}\left(\frac{-\delta z_{N}}{\mathcal{H}(1+z)}\right)^{i} \partial_{r}^{i} \delta\right)\left(1-\frac{d \delta z_{N}}{d z}\right)-1= \\
& =-\frac{d \delta z_{N}}{d z}+\sum_{i=0} \frac{1}{i !}\left(\frac{-\delta z_{N}}{\mathcal{H}(1+z)}\right)^{i} \partial_{r}^{i} \delta\left(1-\frac{d \delta z_{N}}{d z}\right)= \\
& =\sum_{i=0} \frac{\mathcal{H}^{-i}}{i !} \partial_{r}^{i} v_{\|}^{i}-1+\sum_{i=0} \frac{1}{i !}\left(\frac{-\delta z_{N}}{\mathcal{H}(1+z)}\right)^{i} \partial_{r}^{i} \delta\left(1-\frac{d \delta z_{N}}{d z}\right)
\end{aligned}
$$

we remark that we just need to show

$$
\sum_{n=0} \frac{\mathcal{H}^{-n+1}}{(n-1) !} \partial_{r}^{n-1}\left(\delta v_{\|}^{n-1}\right)=\sum_{i=0} \frac{1}{i !}\left(\frac{-\delta z_{N}}{\mathcal{H}(1+z)}\right)^{i} \partial_{r}^{i} \delta\left(1-\frac{d \delta z_{N}}{d z}\right)-1
$$

By plugging in the redshift perturbation (6.3), the right-hand side reads as

$$
\begin{aligned}
& \sum_{i=0} \frac{1}{i !}\left(\frac{-\delta z_{N}}{\mathcal{H}(1+z)}\right)^{i} \partial_{r}^{i} \delta\left(1-\frac{d \delta z_{N}}{d z}\right)-1= \\
& =\sum_{i=0} \frac{1}{i !}\left(\sum_{\ell=0} \frac{\mathcal{H}^{-\ell-1}}{(1+\ell) !} \partial_{r}^{\ell} v_{\|}^{\ell+1}\right)^{i} \partial_{r}^{i} \delta\left(\sum_{j=0} \frac{\mathcal{H}^{-j}}{j !} \partial^{j} v_{\|}^{j}\right)-1
\end{aligned}
$$




$$
=\sum_{i=0, j=0} \frac{\mathcal{H}^{-j-i}}{i ! j !} \partial_{r}^{i} \delta \partial^{j} v_{\|}^{j}\left(\sum_{\ell=0} \frac{\mathcal{H}^{-\ell}}{(1+\ell) !} \partial_{r}^{\ell} v_{\|}^{\ell+1}\right)^{i}-1 .
$$

We can also rewrite it as a sum over all perturbative orders $n$, like the left-hand side of eq. (8.5). In this case, at a given perturbative order $n$, the internal sum over the index $\ell$ is bounded by $n-1-j$, and therefore we have

$$
\left[\sum_{i=0, j=0} \frac{\mathcal{H}^{-j-i}}{i ! j !} \partial_{r}^{i} \delta \partial^{j} v_{\|}^{j}\left(\sum_{\ell=0}^{n-1-j} \frac{\mathcal{H}^{-\ell}}{(1+\ell) !} \partial_{r}^{\ell} v_{\|}^{\ell+1}\right)^{i}\right]^{(n)}=\sum_{i=0}^{n-1} \sum_{j=0}^{n-i-1} \frac{\mathcal{H}^{-j-i}}{i ! j !} \partial_{r}^{i} \delta \partial^{j} v_{\|}^{j} G_{i j}^{(n-1-j)}
$$

where we have used the multinomial theorem obtaining

$$
\begin{aligned}
& G_{i j}^{(n-1-j)} \equiv\left[\left(\sum_{\ell=0}^{n-1-j} \frac{\mathcal{H}^{-\ell}}{(1+\ell) !} \partial_{r}^{\ell} v_{\|}^{\ell+1}\right)^{i}\right]_{\substack{(n-1-j) \\
k_{0}+. .+k_{n-1}=i \\
\sum_{\ell=0}^{\ell-1}(\ell+1) k_{\ell}=n-1-j}} \frac{i !}{k_{0} ! . . k_{n-1} !} \prod_{t=0}^{n-1}\left(\frac{\mathcal{H}^{-t}}{(t+1) !} \partial_{r}^{t} v_{\|}^{t+1}\right)^{k_{t}} \\
& =\mathcal{H}^{1+j+i-n}\left(\begin{array}{c}
n-j-2 \\
i-1
\end{array}\right) \frac{i !}{(n-j-1) !} \partial_{r}^{n-1-j-i} v_{\|}^{n-1-j} \\
& =\frac{i}{n-j-1} \frac{\mathcal{H}^{1+j+i-n}}{(n-j-i-1) !} \partial_{r}^{n-1-j-i} v_{\|}^{n-1-j} \text {. }
\end{aligned}
$$

So combining with eq. (8.7) we simply get

$$
\begin{aligned}
& {\left[\sum_{i=0, j=0} \frac{\mathcal{H}^{-j-i}}{i ! j !} \partial_{r}^{i} \delta \partial^{j} v_{\|}^{j}\left(\sum_{\ell=0}^{n-1-j} \frac{\mathcal{H}^{-\ell}}{(1+\ell) !} \partial_{r}^{\ell} v_{\|}^{\ell+1}\right)^{i}\right]^{(n)}=} \\
& \quad=\sum_{i=0}^{n-1} \sum_{j=0}^{n-i-1} \frac{\mathcal{H}^{1-n}}{(i-1) ! j !} \frac{1}{(n-j-1)(n-j-i-1) !} \partial_{r}^{i} \delta \partial_{r}^{j} v_{\|}^{j} \partial_{r}^{n-1-j-i} v_{\|}^{n-1-j} .
\end{aligned}
$$

We expand now the left-hand side of eq. (8.5)

$$
\begin{aligned}
\frac{\mathcal{H}^{-n+1}}{(n-1) !} \partial_{r}^{n-1}\left(\delta v_{\|}^{n-1}\right) & =\frac{\mathcal{H}^{-n+1}}{(n-1) !} \sum_{i=0}^{n-1}\left(\begin{array}{c}
n-1 \\
i
\end{array}\right) \partial_{r}^{i} \delta \partial_{r}^{n-1-i} v_{\|}^{n-1} \\
& =\sum_{i=0}^{n-1} \frac{\mathcal{H}^{1-n}}{i !(n-1-i) !} \partial_{r}^{i} \delta \partial_{r}^{n-1-i} v_{\|}^{n-1} .
\end{aligned}
$$

To conclude this proof we use

$$
\partial_{r}^{M} v_{\|}^{N}=\sum_{j=0}^{M} \frac{M-N}{j-N}\left(\begin{array}{c}
M \\
j
\end{array}\right) \partial_{r}^{j} v_{\|}^{j} \partial_{r}^{M-j} v_{\|}^{N-j}
$$

for $N \geq 1$ and $M \leq N-1$. Indeed, we simply need to replace $M=n-i-1$ and $N=n-1$ to proof the validity of eq. (8.5). Therefore, we have formally shown that this approach recovers the standard Newtonian perturbation theory in redshift space to any order in perturbation theory. 


\section{Conclusions}

In this paper we have derived a novel approach to compute the (leading) relativistic corrections to the galaxy number counts to any perturbative order. We fully describe the relativistic effects, see eq. (6.1), in terms of redshift perturbation, eq. (3.15), offering a neat interpretation of the output of relativistic N-body simulations (through geodesic light-tracing [15]). As in N-body simulations, where the angular and redshift positions need to be corrected by the observed incoming photon direction and redshift perturbation by solving the geodesic propagation equations, we can write the relativistic effects in terms of the deflection angle and redshift perturbation. In our work we have focused on the redshift perturbation since it is the main source of the odd multipoles of the power spectrum or correlation function, while the deflection angle is sourced by the well-known lensing effect.

We have shown that this approach agrees up to third order ${ }^{6}$ with previous derivations of relativistic effects. We, for the first time, quantified the impact of the gravitational potential as well as linear and transverse Doppler effects on the galaxy number counts. Moreover, we have proven that the Newtonian contributions are equivalent to standard perturbation theory to any order.

In an accompanying paper [38], we have applied and tested this formalism with relativistic N-body simulations provided by [15].

\section{Acknowledgments}

ED (No. 171494 and 171506) acknowledges financial support from the Swiss National Science Foundation. FB is a Royal Society University Research Fellow.

\section{References}

[1] J. Yoo, A. L. Fitzpatrick, and M. Zaldarriaga, A New Perspective on Galaxy Clustering as a Cosmological Probe: General Relativistic Effects, Phys.Rev. D80 (2009) 083514, [arXiv:0907.0707].

[2] J. Yoo, General relativistic description of the observed galaxy power spectrum: Do we understand what we measure?, Phys.Rev.D 82 (2010) 083508, [arXiv:1009.3021].

[3] C. Bonvin and R. Durrer, What galaxy surveys really measure, Phys.Rev. D84 (2011) 063505, [arXiv:1105.5280].

[4] A. Challinor and A. Lewis, The linear power spectrum of observed source number counts, Phys.Rev. D84 (2011) 043516, [arXiv:1105.5292].

[5] A. Hall, C. Bonvin, and A. Challinor, Testing General Relativity with 21-cm intensity mapping, Phys. Rev. D87 (2013), no. 6 064026, [arXiv:1212.0728].

[6] J. Yoo and M. Zaldarriaga, Beyond the Linear-Order Relativistic Effect in Galaxy Clustering: Second-Order Gauge-Invariant Formalism, Phys. Rev. D90 (2014), no. 2 023513, [arXiv: 1406.4140].

[7] D. Bertacca, R. Maartens, and C. Clarkson, Observed galaxy number counts on the lightcone up to second order: I. Main result, JCAP 1409 (2014), no. 09 037, [arXiv: 1405.4403].

\footnotetext{
${ }^{6}$ Previous derivations have stopped to third order.
} 
[8] D. Bertacca, R. Maartens, and C. Clarkson, Observed galaxy number counts on the lightcone up to second order: II. Derivation, JCAP 11 (2014) 013, [arXiv:1406.0319].

[9] E. Di Dio, R. Durrer, G. Marozzi, and F. Montanari, Galaxy number counts to second order and their bispectrum, JCAP 1412 (2014) 017, [arXiv:1407.0376]. [Erratum: JCAP1506,no.06,E01(2015)].

[10] V. Iršič, E. Di Dio, and M. Viel, Relativistic effects in Lyman- $\alpha$ forest, JCAP 1602 (2016), no. 02 051, [arXiv:1510.03436].

[11] E. Di Dio, F. Montanari, A. Raccanelli, R. Durrer, M. Kamionkowski, and J. Lesgourgues, Curvature constraints from Large Scale Structure, JCAP 1606 (2016), no. 06 013, [arXiv:1603.09073].

[12] E. Di Dio and U. Seljak, The relativistic dipole and gravitational redshift on LSS, JCAP 1904 (2019), no. 04 050, [arXiv: 1811.03054].

[13] C. Clarkson, E. M. de Weerd, S. Jolicoeur, R. Maartens, and O. Umeh, The dipole of the galaxy bispectrum, Mon. Not. Roy. Astron. Soc. 486 (2019), no. 1 L101-L104, [arXiv:1812.09512].

[14] R. Maartens, S. Jolicoeur, O. Umeh, E. M. De Weerd, C. Clarkson, and S. Camera, Detecting the relativistic galaxy bispectrum, JCAP 03 (2020), no. 03 065, [arXiv: 1911.02398].

[15] M.-A. Breton, Y. Rasera, A. Taruya, O. Lacombe, and S. Saga, Imprints of relativistic effects on the asymmetry of the halo cross-correlation function: from linear to non-linear scales, Mon. Not. Roy. Astron. Soc. 483 (2019), no. 2 2671-2696, [arXiv:1803.04294].

[16] A. Lewis and A. Challinor, Weak gravitational lensing of the cmb, Phys. Rept. 429 (2006) 1-65, [astro-ph/0601594].

[17] M. Bartelmann and P. Schneider, Weak gravitational lensing, Phys. Rept. 340 (2001) 291-472, [astro-ph/9912508].

[18] P. Schneider, Weak gravitational lensing, in Proceedings, 33rd Advanced Saas Fee Course on Gravitational Lensing: Strong, Weak, and Micro: Les Diablerets, Switzerland, April 7-12, 2003, pp. 269-451, 2006. astro-ph/0509252.

[19] M. Kilbinger, Cosmology with cosmic shear observations: a review, Rept. Prog. Phys. $\mathbf{7 8}$ (2015) 086901, [arXiv:1411.0115].

[20] E. Schaan, S. Ferraro, and D. N. Spergel, Weak Lensing of Intensity Mapping: the Cosmic Infrared Background, Phys. Rev. D97 (2018), no. 12 123539, [arXiv:1802.05706].

[21] K. S. Mandel and M. Zaldarriaga, Weak gravitational lensing of high-redshift $21 \mathrm{~cm}$ power spectra, Astrophys. J. 647 (2006) 719-736, [astro-ph/0512218].

[22] L. Hui, E. Gaztanaga, and M. LoVerde, Anisotropic Magnification Distortion of the 3D Galaxy Correlation. 1. Real Space, Phys. Rev. D76 (2007) 103502, [arXiv:0706.1071].

[23] M. LoVerde, L. Hui, and E. Gaztanaga, Lensing corrections to features in the angular two-point correlation function and power spectrum, Phys. Rev. D77 (2008) 023512, [arXiv: 0708.0031].

[24] L. Hui, E. Gaztanaga, and M. LoVerde, Anisotropic Magnification Distortion of the 3D Galaxy Correlation: II. Fourier and Redshift Space, Phys. Rev. D77 (2008) 063526, [arXiv:0710.4191].

[25] S. Dodelson, F. Schmidt, and A. Vallinotto, Universal Weak Lensing Distortion of Cosmological Correlation Functions, Phys. Rev. D78 (2008) 043508, [arXiv:0806.0331]. 
[26] F. Montanari and R. Durrer, Measuring the lensing potential with tomographic galaxy number counts, JCAP 1510 (2015), no. 10 070, [arXiv:1506.01369].

[27] E. Di Dio, Lensing smoothing of BAO wiggles, JCAP 1703 (2017), no. 03 016, [arXiv: 1609.09044].

[28] N. Kaiser, Clustering in real space and in redshift space, Mon. Not. Roy. Astron. Soc. 227 (1987) 1-27.

[29] P. McDonald, Gravitational redshift and other redshift-space distortions of the imaginary part of the power spectrum, JCAP 0911 (2009) 026, [arXiv:0907.5220].

[30] J. Yoo, N. Hamaus, U. Seljak, and M. Zaldarriaga, Testing general relativity on horizon scales and the primordial non-gaussianity, Phys.Rev.D 86 (2012) 063514, [arXiv:1109.0998].

[31] C. Bonvin, L. Hui, and E. Gaztanaga, Asymmetric galaxy correlation functions, Phys. Rev. D89 (2014), no. 8 083535, [arXiv:1309.1321].

[32] E. Gaztanaga, C. Bonvin, and L. Hui, Measurement of the dipole in the cross-correlation function of galaxies, JCAP 1701 (2017), no. 01 032, [arXiv:1512.03918].

[33] C. Bonvin, L. Hui, and E. Gaztanaga, Optimising the measurement of relativistic distortions in large-scale structure, JCAP 1608 (2016), no. 08 021, [arXiv:1512.03566].

[34] A. Hall and C. Bonvin, Measuring cosmic velocities with $21 \mathrm{~cm}$ intensity mapping and galaxy redshift survey cross-correlation dipoles, Phys. Rev. D95 (2017), no. 4 043530, [arXiv: 1609.09252].

[35] C. Bonvin, S. Andrianomena, D. Bacon, C. Clarkson, R. Maartens, T. Moloi, and P. Bull, Dipolar modulation in the size of galaxies: The effect of Doppler magnification, Mon. Not. Roy. Astron. Soc. 472 (2017), no. 4 3936-3951, [arXiv:1610.05946].

[36] E. Giusarma, S. Alam, H. Zhu, R. A. C. Croft, and S. Ho, Relativistic asymmetries in the galaxy cross-correlation function, arXiv:1709.07854.

[37] F. Lepori, E. Di Dio, E. Villa, and M. Viel, Optimal galaxy survey for detecting the dipole in the cross-correlation with $21 \mathrm{~cm}$ Intensity Mapping, JCAP 1805 (2018), no. 05043 , [arXiv:1709.03523].

[38] F. Beutler and E. Di Dio, Modeling relativistic contributions to the halo power spectrum dipole, JCAP 07 (2020), no. 07 048, [arXiv: 2004.08014].

[39] A. Raccanelli, D. Bertacca, R. Maartens, C. Clarkson, and O. Doré, Lensing and time-delay contributions to galaxy correlations, Gen. Rel. Grav. 48 (2016), no. 7 84, [arXiv: 1311.6813].

[40] D. Alonso, P. Bull, P. G. Ferreira, R. Maartens, and M. Santos, Ultra large-scale cosmology in next-generation experiments with single tracers, Astrophys. J. 814 (2015), no. 2 145, [arXiv:1505.07596].

[41] A. Raccanelli, F. Montanari, D. Bertacca, O. Doré, and R. Durrer, Cosmological Measurements with General Relativistic Galaxy Correlations, JCAP 1605 (2016), no. 05 009, [arXiv:1505.06179].

[42] W. Cardona, R. Durrer, M. Kunz, and F. Montanari, Lensing convergence and the neutrino mass scale in galaxy redshift surveys, Phys. Rev. D94 (2016), no. 4 043007, [arXiv:1603.06481].

[43] C. S. Lorenz, D. Alonso, and P. G. Ferreira, Impact of relativistic effects on cosmological parameter estimation, Phys. Rev. D97 (2018), no. 2 023537, [arXiv:1710.02477]. 
[44] E. Villa, E. Di Dio, and F. Lepori, Lensing convergence in galaxy clustering in CDM and beyond, JCAP 1804 (2018), no. 04 033, [arXiv: 1711.07466].

[45] C. Bonvin and P. Fleury, Testing the equivalence principle on cosmological scales, JCAP 1805 (2018), no. 05 061, [arXiv: 1803.02771].

[46] S. R. Green and R. M. Wald, A new framework for analyzing the effects of small scale inhomogeneities in cosmology, Phys. Rev. D 83 (2011) 084020, [arXiv:1011.4920].

[47] S. R. Green and R. M. Wald, Newtonian and Relativistic Cosmologies, Phys. Rev. D 85 (2012) 063512, [arXiv:1111.2997].

[48] J. Adamek, D. Daverio, R. Durrer, and M. Kunz, General Relativistic N-body simulations in the weak field limit, Phys. Rev. D 88 (2013), no. 10 103527, [arXiv:1308.6524].

[49] J. Adamek, C. Clarkson, R. Durrer, and M. Kunz, Does small scale structure significantly affect cosmological dynamics?, Phys. Rev. Lett. 114 (2015), no. 5 051302, [arXiv: 1408.2741].

[50] J. Adamek, D. Daverio, R. Durrer, and M. Kunz, gevolution: a cosmological N-body code based on General Relativity, JCAP 07 (2016) 053, [arXiv: 1604.06065].

[51] C. Fidler, T. Tram, C. Rampf, R. Crittenden, K. Koyama, and D. Wands, General relativistic weak-field limit and Newtonian N-body simulations, JCAP 12 (2017) 022, [arXiv: 1708.07769].

[52] L. Castiblanco, R. Gannouji, J. Noreña, and C. Stahl, Relativistic cosmological large scale structures at one-loop, JCAP 07 (2019) 030, [arXiv:1811.05452].

[53] E. Di Dio, F. Montanari, J. Lesgourgues, and R. Durrer, The CLASSgal code for Relativistic Cosmological Large Scale Structure, JCAP 1311 (2013) 044, [arXiv:1307.1459].

[54] T. Baldauf, U. Seljak, L. Senatore, and M. Zaldarriaga, Galaxy Bias and non-Linear Structure Formation in General Relativity, JCAP 10 (2011) 031, [arXiv:1106.5507].

[55] D. Jeong, F. Schmidt, and C. M. Hirata, Large-scale clustering of galaxies in general relativity, Phys. Rev. D 85 (2012) 023504, [arXiv:1107.5427].

[56] Z. Vlah, U. Seljak, P. McDonald, T. Okumura, and T. Baldauf, Distribution function approach to redshift space distortions. Part IV: perturbation theory applied to dark matter, JCAP 1211 (2012) 009, [arXiv:1207.0839]. 